\title{
À la recherche de la nature et de sa vision aux Samoa Américaines
}

\section{Marieke Blondet}

\section{(2) OpenEdition}

1 Journals

\section{Édition électronique}

URL : http://journals.openedition.org/jso/5422

DOI : $10.4000 /$ jso. 5422

ISSN : $1760-7256$

\section{Éditeur}

Société des océanistes

\section{Édition imprimée}

Date de publication : 15 décembre 2008

Pagination : 127-144

ISBN : 978-2-85430-012-3

ISSN : 0300-953x

Référence électronique

Marieke Blondet, "À la recherche de la nature et de sa vision aux Samoa Américaines », Journal de la Société des Océanistes [En ligne], 126-127 | Année 2008, mis en ligne le 15 décembre 2011, consulté le 17 juin 2020. URL : http://journals.openedition.org/jso/5422 ; DOl : https://doi.org/10.4000/jso.5422 


\section{À la recherche de la nature et de sa vision aux Samoa Américaines ${ }^{1}$}

par

Marieke BLONDET*

\section{RÉSUMÉ}

Selon la littérature anthropologique, les Polynésiens, notamment les Samoans américains, ont une relation étroite avec leur environnement naturel tant leur vie matérielle et idéelle dépend d'éléments puisés dans la nature. Cependant, les Samoa américains ont subi, depuis plus d'un siècle, l'influence de la culture nordaméricaine et ont abandonné nombre d'activités traditionnelles provoquant une rupture du lien qui, jusque-là, unissait l'homme à la nature. Aujourd'hui la population de ces îles a perdu la relation étroite qu'elle avait avec son environnement et ses îles connaissent une très forte pollution d'origine anthropique. Après avoir évoqué l'histoire coloniale de ces îles et la relation ancestrale qu'elles abritaient entre les hommes et la nature, l'article présentera l'état de l'environnement des Samoa américaines et montrera que, malgré l'existence de programmes d'éducation et la création d'un parc national, la protection de l'environnement est un échec. Pour finir, je proposerai des pistes de réflexion pour expliquer cet état des choses, faisant notamment appel à la vision du monde des Samoans et à leur rapport à la terre.

Mots-CLÉs : environnement, terre, cosmologie, américanisation, système matai

En Polynésie, la terre a toujours été au centre de l'existence des habitants des îles, tant parce

\begin{abstract}
According to the anthropologic literature, people in Polynesia, in particular American Samoans, have a very strong relationship with their nature as much of their material and symbolic life rely on natural elements. However, American Samoa are under the northamerican influence for more than a century now. Under this influence many traditional activities have progressively disappeared and the link between men and nature came apart. Moreover the islands environment is affected by severe human pollutions as the men feel more and more distant from nature. In this paper I remind the colonial history of American Samoa and the traditional relationship existing there between men and nature. Then I show that even under the benefit of several educational programs and the existence, among others, of a national park, the conservation of nature is unsuccessful. Finally I propose two possible answers to that issue: an analysis of the Samoan people behavior through their vision of the world and through their relationship to their land.
\end{abstract}

KeYWORDS: environment, land, cosmology, americanization, matai system

qu'ils tiraient de leur environnement naturel l'ensemble de leurs moyens de subsistance, que

1. Cet article est le fruit d'une recherche de terrain réalisée entre 2006 et 2007 dans le cadre d'un travail de doctorat. Les questions qui y sont traitées constituent une partie de la thèse à venir et sont parfois présentées à leur stade préliminaire.

* Doctorante EHESS - IRIS (UMR 8156 - 723 INSERM - UP 13) en cotutelle avec l'université de Otago (Nouvelle-Zélande), cookiedo@hotmail.fr 
parce que cette relation des hommes à leur terre déterminait la place de chacun au sein de la communauté. Les Samoa américaines, il y a encore quelques décennies, ne dérogeaient pas à cette règle. Pourtant, on observe, aujourd'hui, qu'une distance se creuse entre les hommes et la nature, cette dernière souffrant de plus en plus de pollution, de surexploitation et d'un manque évident d'attention de la part de la population. Le lien entre les hommes et la nature semble rompu. Une explication communément avancée dans le Pacifique pour rendre compte des actes de pollution veut, qu'autrefois, les habitants des îles se servaient exclusivement d'objets et d'aliments naturels et qu'après utilisation, ou lorsque ces objets étaient endommagés, ils les rejetaient dans la nature où ils finissaient par disparaître. Aujourd'hui, les îliens continueraient à agir de la sorte et à rejeter les objets usagés, mais, hélas, ceux-ci ne sont plus biodégradables comme avant (Turner, 2005 : 9). Si bien sûr il est essentiel que ces populations comprennent ce que leur attitude a de nuisible pour l'environnement, l'interprétation présentée ci-dessus me semble cependant un peu simple et réductrice. Il me paraît plus juste, pour expliquer cet état de fait, de se demander si la notion de préservation de la nature telle qu'elle est promue par l'Occident a une signification dans des sociétés non occidentales comme, ici, celle des îles Samoa (Leblic, 2005 : 108). Cette indifférence des îliens vis-à-vis de recommandations occidentales pourrait bien être une des explications possibles de l'échec des programmes d'éducation aux questions d'environnement introduits dans ce pays. Je vais présenter quelques-unes des réponses apportées à ces interrogations dans le cas des Samoa américaines.

Après un rappel de l'histoire coloniale de ce petit État pour mieux saisir sa situation actuelle, notamment la relation à la nature qu'entretenait jadis sa population, je présenterai les conditions de l'environnement naturel des îles. J'évoquerai les tentatives de protection de la nature ainsi que leur échec relatif pour infléchir les habitudes polluantes des Samoans américains. De là, je discuterai de ce qu'est, aujourd'hui, l'attitude de ceux-ci envers leur nature et de la manière dont ils la perçoivent et la mettent en pratique. Enfin, je proposerai deux pistes de réflexion sur le fait que, malgré la qualité de la relation ancestrale des Samoans avec leur nature et les nombreux programmes de protection de celle-ci qui leur ont été proposés, la conservation de celle-ci soit un échec. D'une part, je suggérerai que cette attitude repose sur une incompréhension entre différentes visions du monde, l'une écologiste occidentale, l'autre samoane et, d'autre part, je traiterai des récentes évolutions de cette société samoane, notamment dans son rapport à la terre. Ces deux analyses permettent, selon moi, une meilleure compréhension de l'attitude psychologique des Samoans vis-à-vis de leur environnement et pourraient être à même, dans le futur, d'aider à une meilleure protection de celui-ci.

\section{Présentation des Samoa américaines}

Les Samoa américaines sont un petit archipel de $199 \mathrm{~km}^{2}$ situé à l'est des Samoa indépendantes ${ }^{2}$. Il est constitué de cinq îles volcaniques, Tutuila, Aunu'u, Ofu, Olosega et Ta'u, et de deux atolls, Rose atoll et Swain island. Tutuila, la plus grande des îles $\left(150 \mathrm{~km}^{2}\right)$, concentre à elle seule $90 \%$ de la population (Craig, 2005). Le territoire est affilié aux États-Unis, mais jouit d'une autonomie certaine et possède sa propre constitution et son propre gouvernement avec, à sa tête, un gouverneur élu. Il doit cependant se plier à la législation américaine dans certains domaines juridiques, notamment en matière de protection de l'environnement ${ }^{3}$. Les habitants ne sont pas citoyens américains, mais "nationaux »; ils profitent des mêmes avantages diplomatiques et sociaux que ceux-ci, notamment de la possession d'un passeport américain, mais ne peuvent pas voter lors des élections fédérales américaines.

Ce statut original est le résultat de l'histoire coloniale américaine. Dans leur course au marché chinois, durant la seconde moitié du $\mathrm{XIX}^{\mathrm{e}}$ siècle, les Allemands, les Américains et les Anglais entrèrent en compétition auprès des populations des Samoa, pour y établir des dépôts de charbon nécessaires au ravitaillement de leurs bateaux. Pago Pago, la capitale des Samoa américaines, était alors l'un des ports les plus sûrs de la région (Heffer, 1995). Alors qu'à l'époque, les Samoa et les Samoa américaines ne formaient qu'une seule entité, la rivalité entre les

2. J'emploie ici le terme de «Samoa indépendantes» pour marquer la différence avec les Samoa américaines, mais les habitants du premier État, ainsi que leurs dirigeants, revendiquent le nom simple de « Samoa », sans marquer de distinction, ce que je respecterai dans la suite du texte.

3. Plusieurs agences fédérales américaines en charge de questions relatives à l'environnement sont présentes aux Samoa américaines : l'Environmental Protection Agency (EPA), le National Park Service (NPS) dont l'action s'étend d'ailleurs au-delà du parc national ou encore la National Oceanic and Atmospheric Administration (NOAA). 


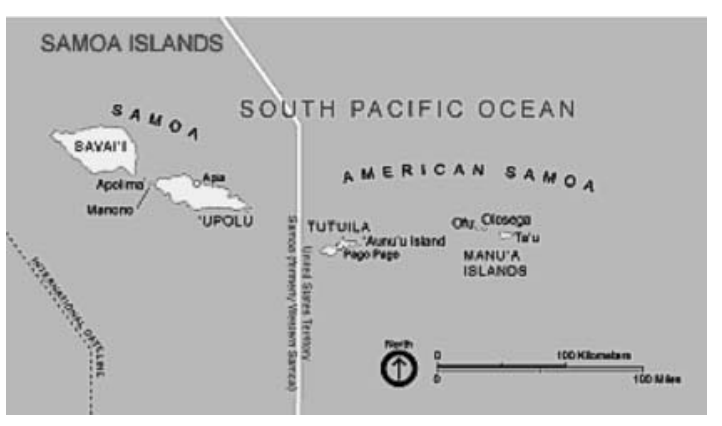

Figure 1. - Carte des deux Samoa

trois puissances les amena à intervenir dans la politique des îles et, finalement, à s'en partager le contrôle, lors de la signature du traité de Berlin en 1889: les Allemands obtinrent l'actuel Samoa, les Américains les Samoa américaines et les Anglais les îles Marshall et des territoires en Afrique (Gray, 1980).

Les Américains confièrent la gestion des îles ainsi acquises à leur Navy et, compte tenu du peu de ressources naturelles exploitables, intervinrent peu et laissèrent les habitants vivre comme ils l'avaient fait jusque-là. L'influence américaine commença réellement à se faire sentir lors du conflit avec le Japon, quand de nombreux militaires stationnèrent sur l'île de Tutuila. Si la guerre en elle-même affecta peu les îles, la présence militaire et l'économie de marché qu'elle y induisit eurent, sur elles, un effet dévastateur. Les Américains développèrent les équipements $\mathrm{du}$ petit territoire (routes, aéroport, hôpital, réseaux électriques) et, une fois la guerre finie et la base militaire démantelée, le territoire se trouva doté d'infrastructures impossibles à maintenir par ses seuls moyens. De nouveaux besoins avaient aussi été créés dans la population qui, ayant goûté à l'American way of life et au confort des emplois salariés, n'avait plus envie de retourner au dur labeur des champs de taro ou des activités de pêche (Gray, 1980; Shaffer, 2000). Pour venir en aide à leur territoire, les États-Unis décidèrent d'une aide financière annuelle qui perdure depuis lors. Si aujourd'hui les Samoa américaines possèdent une autonomie certaine et un niveau de développement supérieur à celui des îles voisines, elles sont toujours très dépendantes de l'aide fédérale qui représente la moitié de son budget annuel. La deuxième moitié provient de la seule industrie des îles, deux conserveries de thon, implantées dans les années 1950-1960 (American Samoa's Comprehensive Economic Development Strategy, 2005). Aujourd'hui, l'économie du territoire est limitée. Les conserveries menacent de partir pour des contrées plus accueillantes en termes de coûts de production et d'exportation. L'industrie touristique, qui avait débuté avec succès dans les années 1970, végète depuis lors. Corrélativement, l'émigration des Samoans américains s'est accélérée. Elle avait débuté dans les années 1950 avec une première vague de migrants invités par le gouvernement fédéral américain à venir tenter leur chance aux États-Unis, principalement à Hawaii et en Californie. Les Samoa américaines ont ainsi connu un passage extrêmement rapide d'une économie traditionnelle à une économie de marché «à l'américaine ». Aujourd'hui, seulement $6 \%$ de la population active continue à pratiquer une activité de subsistance traditionnelle. Ces quelques producteurs fournissent les marchés de l'île où le reste de la population s'approvisionne en produits aussi banals que les taros, les bananes et les noix de coco. Le poisson a cessé d'être consommé de manière régulière ; on l'achète au supermarché. Quelques personnes continuent pourtant de cultiver, de temps en temps, leurs champs de taro ou de pêcher pour le profit de leur famille immédiate ou par plaisir, faisant de cette activité un loisir. Dans le village où j'ai séjourné, une quinquagénaire m'expliquait qu'elle appréciait particulièrement d'aller passer une nuit sur sa plantation située sur les pentes de la montagne, derrière le village, non pour travailler la terre de sa famille, mais pour y profiter du calme et de la douceur de l'air. Aujourd'hui, aux Samoa américaines, on va sur sa plantation comme on va dans une « campagne » dans les pays occidentaux.

Que nous apprennent ces quelques observations sur l'attitude des Samoans envers leur environnement ? À la suite du passage de leur population à une société de consommation, renforcé par des contacts intensifs avec les diasporas hawaiienne et californienne, les Samoans américains ont adopté de nouvelles habitudes de vie et recherchent de nombreux biens de facture occidentale dont ils n'avaient pas l'utilité auparavant (assiettes et couverts en plastique, boîtes en polystyrène pour transporter de la nourriture, canettes de soda, voitures $4 \times 4$ ). À l'évidence, ces biens sont aujourd'hui les signes d'un statut économique, si ce n'est social, supérieur. Ceci, associé à l'augmentation continue de la population ${ }^{4}$, fait peser une pression très forte sur l'environnement naturel, comme nous le verrons plus loin. En parallèle, on assiste à une désaffection générale pour les activités de plein air. Si les gens allaient autrefois marcher en forêt pour s'appro-

4. Avant 1990 , le taux de croissance de la population était supérieur à $+4 \%$ par an. Il a depuis décru pour aujourd'hui atteindre $+2 \%$ par an, mais reste très fort en comparaison avec les îles voisines. 
visionner en bois de construction et en plantes médicinales, ils restent aujourd'hui chez eux et regardent les programmes américains à la télévision ou des films en DVD. On ne marche pas non plus pour aller travailler, on prend sa voiture pour se rendre sur son lieu de travail situé de l'autre côté de l'île. De même, les enfants ne vont plus à l'école à pied. Dans le village de 650 habitants où je résidais et où l'enfant le plus éloigné est à moins de 10 minutes de marche de son école, il existe un service de bus scolaire semblable à celui qui fait le paysage des petites villes américaines. Le lien avec la nature, qui était entretenu par ces activités d'extérieur, a peu à peu été rompu. Pourtant, ce qui frappe, c'est l'énorme littérature, anthropologique ou autre, qui a décrit la forte connexion ancestrale des gens des îles du Pacifique avec leurs terres de famille et, au-delà, avec les éléments naturels propres à ces terres. Cette relation suscitait, jadis, un grand respect des populations pour leurs terres et la nature en général et les incitait à en assurer la protection. Ce n'est plus le cas aux Samoa américaines. Dans quelle situation se trouve l'environnement naturel de ces îles?

\section{Situation de l'environnement et programmes de protection de la nature}

Nous venons de le rappeler, l'augmentation de la population et son adoption de biens de consommation manufacturés pèsent de plus en plus fort sur l'environnement des Samoa américaines, surtout celui de Tutuila, la plus grande et la plus peuplée des îles. Face à la poussée démographique, on cherche à gagner du terrain sur la mer ou on déforeste la montagne au risque de provoquer des glissements de terrain en saison des pluies. Des problèmes de consommation d'énergie (essence, gaz) et d'eau potable ainsi que de gestion des objets usagés (piles, boîtes de conserve, sacs en plastiques) se posent. Un dernier problème s'ajoute encore, celui de la pollution industrielle, qui affecte, depuis plusieurs décennies, le port de Pago Pago. Celui-ci souffre non seulement du rejet dans la mer des déchets des conserveries, mais aussi des dégazages illégaux et du rejet, par les bateaux de pêche venus approvisionner les usines, de leurs eaux usagées (Holmes and Holmes, 1992 : 126). En 1990, un bulletin d'alerte de l'Environmental Protection Agency interdisait la consommation des poissons pêchés dans le port et y décourageait la baignade. Et si, en 1991, les conserveries furent sommées de mettre fin à leurs déversements dans le port, elles continuent de rejeter leurs déchets ... en haute mer. Aujourd'hui, l'interdiction de pêche est toujours en vigueur et l'état de l'environnement en général reste particulièrement critique. De nombreux projets d'éducation ont cependant été mis en place depuis plus de vingt ans par des organismes gouvernementaux locaux ou dépendant du département de l'Intérieur des États-Unis. Ces programmes cherchent à faire prendre conscience à la population des menaces que les activités humaines, telles que le rejet d'objets usagés dans la nature, les déchets des élevages de cochons ou encore l'utilisation de pesticides en agriculture et de poison ou de dynamite pour la pêche, font peser sur l'environnement. Des manifestations destinées à promouvoir la sauvegarde de l'environnement sont régulièrement organisées. En 2006, par exemple, le gouvernement des Samoa américaines a participé à "l'Année de la tortue de mer", dans le cadre d'un programme international à l'initiative de l'oISEA ${ }^{5}$ pour protéger cet animal dans l'ensemble des îles du Pacifique Sud. Il existe aussi sur le territoire plusieurs réserves naturelles tournées vers la protection du récif corallien et des ressources marines comme le Fagatele Bay National Marine Sanctuary. En ce qui concerne l'environnement naturel terrestre, enfin, on ne trouve aujourd'hui qu'un seul organisme ayant une telle vocation, un parc national. Celui-ci a été créé par le gouvernement fédéral américain en 1993 pour protéger la forêt paléo-tropicale, une partie du récif corallien et des éléments du patrimoine culturel samoan puisque l'on trouve, au cœur du parc, certains des sites archéologiques les plus riches et les plus importants de ces îles (Butcher, 1999 : 82 ; Craig, 2005 : 10-11).

\section{Le Parc national des Samoa américaines}

À la fin des années 1980 et face à la disparition des Samoan Flying Fox et White Collared Flying Fox, deux espèces de chauves-souris tonganes, un groupe d'activistes écologistes américains réussit à convaincre le gouverneur des Samoa américaines de l'importance de créer un parc naturel pour protéger ces espèces et leur habitat. Ces chauves-souris frugivores participent en effet à la pollinisation des espèces forestières par la dispersion des graines des fruits qu'elles consomment. En 1988, sous la pression de ces écologistes, le Congrès américain signa un acte (Public Law 100-571, October 31, 1988) autori-

5. « Océan Indien et Sud Est asiatique » est une organisation internationale qui agit pour la protection des fonds marins dans les régions de l'océan Indien et du Pacifique Sud. 
sant l'ouverture du National Park of American Samoa (NPAS), rattaché au réseau des parcs nationaux américains et géré par lui. La situation de ce parc est singulière puisque, aux Samoa américaines, le système de fermage foncier est encore majoritairement communautaire : près de $90 \%$ des terres sont toujours aux mains des aiga, les familles étendues samoanes, et la vente de ces terres est interdite à tout non-Samoan. Par conséquent, faute de pouvoir acheter les terres qu'il protège, le gouvernement fédéral américain a dû recourir à un système de location des zones préservées. Le National Park Service (NPS) américain a signé avec les familles de sept villages possédant des terres à l'intérieur du parc, un contrat de location d'une période de cinquante ans. Chaque conseil de village concerné négocia pour son compte le calcul et la répartition des loyers : certains choisirent de répartir la somme totale à parts égales entre chaque aiga, d'autres préférèrent calculer les montants en fonction des surfaces de terre possédées par chaque famille. Une fois dans l'aiga, l'argent est géré par le senior matai, le chef de famille, qui est chargé de la gestion des biens familiaux, principalement des terres communautaires. Il est aussi responsable de la redistribution, entre les membres de sa famille, des profits provenant du travail de ces terres (O'Meara, $1990: 141$; Grattan, $1985: 13$ ) ou, dans le cas présent, l'argent des loyers du parc. En échange du versement de ce loyer, les habitants des villages s'engagent à restreindre certaines de leurs activités à l'intérieur de l'espace protégé. Par exemple, il leur est interdit de défricher de nouvelles parcelles pour étendre les champs de taro, seules les plantations existant avant la création du parc peuvent continuer à être exploitées. La coupe de bois pour la construction d'habitations est aussi interdite et la pêche ne doit se pratiquer qu'avec des techniques traditionnelles (NPAS General Management Plan, 1997 : 22). Mais, compte tenu de l'évolution, ces soixante dernières années, de la société des Samoa américaines, ces restrictions perturbent bien peu la vie des villageois qui ne semblent pas souffrir de l'installation du parc national. Pourtant, cette réserve pourrait avoir une influence positive sur la prise de conscience, par les Samoans américains, de la détérioration de leur environnement.

Le fait que des populations vivent à l'intérieur des limites du parc est une situation inédite dans le réseau des parcs nationaux américains. Aux États-Unis, depuis 1872 et la création du premier parc national américain, celui de Yellowstone, un parc national est, par définition, un espace vide de toute population humaine. Les hommes peuvent venir apprécier ces espaces pendant quelques heures, voire y passer une nuit dans des endroits très circonscrits, mais pas y vivre à plein temps. En toile de fond de cette position, on trouve l'idéologie des tout premiers écologistes américains du $\mathrm{XIX}^{\mathrm{e}}$ siècle pour qui la nature est forcément contaminée par l'homme. Pour ces écologistes de la première heure, la nature, profondément bonne, s'oppose à la civilisation évidemment mauvaise (Wilson, 1992 : 39-41). Aux Samoa américaines, cependant, le NPS n'a pas eu d'autre choix, pour implanter le NPAS, que de conserver à l'intérieur de ses frontières les villages qui existaient là avant l'installation du parc. Cette décision a été motivée, d'une part, par le manque de larges espaces vides et faciles d'accès disponibles pour implanter le parc et, d'autre part, à cause de l'existence du système foncier de fermage si particulier aux Samoa américaines. Enfin, une dernière variable peut expliquer cette décision : le NPS aurait eu la volonté d'intégrer des communautés locales à son projet de parc, suivant en cela les récentes avancées des théories écologiques et de développement qui prônent l'association des populations autochtones aux projets de protection de la nature.

\section{Conservation et populations indigènes : l'état des discussions}

La question du traitement réservé aux autochtones des régions où ont été implantés des parcs et réserves naturels et de leur intégration aux projets d'installation et de gestion, se pose depuis les années 1970. Cependant, ce n'est que depuis les années 1990, sous la pression des luttes autochtones pour la reconnaissance de leurs droits, que les organisations internationales de développement et de protection de la nature (World Bank, UNDP, UNRIST, UNEP) commencèrent à réagir (Wells and Brandon, 1992). Jusquelà, les communautés qui vivaient à l'endroit de l'implantation d'un parc ou d'une réserve, avaient été peu prises en compte dans les projets et, ni leurs besoins, ni l'utilisation pour leur subsistance des ressources naturelles situées à l'intérieur des réserves, n'avaient été comptabilisés. $\mathrm{La}$ plupart du temps, au contraire, ces petites populations étaient traitées comme des bénéficiaires passifs, voire comme une menace pour l'environnement (ibid.). Elles avaient généralement été repoussées à la périphérie du parc et s'étaient vu restreindre certains de leurs droits, comme celui de chasse. Au pire, elles avaient été expulsées hors de leurs terres ancestrales, ou bien les avaient conservées à condition de maintenir leur style de vie traditionnel pour être utilisées 
comme attractions pour les touristes (Colchester, 1994 : 15). De plus en plus de conflits virent donc le jour entre les communautés locales, les gestionnaires des parcs et les gouvernements des pays concernés. C'est dans ce contexte de reconceptualisation des réserves et parcs naturels que le NPAS fut créé.

\section{L'écologie : une idéologie occidentale}

Ces débats autour de l'intégration des « peuples indigènes » aux projets de protection de la nature attirèrent également l'attention sur le fait qu'associer ces populations à la conservation de leur environnement revenait à leur prêter, de facto, des représentations et des préoccupations écologistes occidentales qui leur étaient étrangères. Développant cette idée, Kay Milton explique qu'avoir conscience de la nécessité de protéger l'environnement contre les effets dévastateurs de l'activité humaine est une caractéristique de ce qu'elle appelle «l'environnementalisme ». Cette théorie est, selon elle, symptomatique des sociétés industrielles occidentales, bien qu'elle reconnaisse qu'il existe aussi une certaine forme d'environnementalisme dans les sociétés non occidentales (Milton, 1996 : 27-28). Avec la mondialisation, la conception occidentale de la nature, sauvage et fragile, qu'il faut contrôler pour la protéger, a largement été diffusée notamment au travers du système d'aide au développement mis en place par les organisations internationales et les ONG de protection de l'environnement. Pour qu'un projet de développement reçoive une aide technique et financière de ces organismes, les pays en voie de développement sont contraints de s'engager à respecter les critères occidentaux de conservation et leur mise en œuvre sur le terrain. Certains auteurs parlent même à ce propos de "nouvelle forme de colonialisme" (Sogge, 2004). D'autres proposent qu'au lieu d'appliquer des concepts occidentaux à des contextes qui ne le sont pas, on aille chercher des pratiques de gestion de ces parcs dans la vision du monde de ces peuples autochtones, et que l'on tienne le plus grand cas, en particulier, de la perception qu'ils ont de leur nature. L'objectif est de conférer ainsi une plus grande chance de réussite aux projets de protection de la nature et de développement. L'idée est donc d'intégrer toujours plus les communautés locales, de leur donner davantage de responsabilités dans la mise en place et la gestion des projets et, surtout, d'utiliser les connaissances qu'elles ont de leur terrain (Bryant-Tokalau, 2004; Huffer and Qalo, 2004 ; Overton, 1999 ; Scheyvens and Purdie, 1999). À première vue, les fondateurs du NPAS semblent avoir adopté ce point de vue en intégrant des familles samoanes au projet de parc. Pourtant, dans la réalité, les villageois n'ont pas réellement été impliqués. Les connaissances scientifiques et idéologiques des gestionnaires américains n'ont en rien été croisées avec les savoirs, les techniques et les pratiques des Samoans dans leur cadre naturel. Au final, cette situation aboutit à un demi-échec du parc puisque les populations, n'ayant pas été investies de responsabilités dans le projet, ne se sont pas senties concernées par l'objectif recherché de protection de l'environnement ${ }^{6}$. En somme, malgré l'incorporation des villageois samoans au projet du NPAS qui, on peut le penser, répond à l'évolution des théories et des pratiques écologistes occidentales, cette intégration n'a été que physique mais nullement "participative". Au-delà des limites du parc, la population de Tutuila dans son ensemble n'a nullement été mobilisée et incitée à porter attention et soin à la nature du parc et de l'île dans son ensemble.

Il est essentiel de rechercher la cause de cet échec. Si l'on se tourne du côté américain, deux questions, simples, se posent : les Samoans sontils imperméables à l'idéologie écologiste occidentale véhiculée par le Service des parcs nationaux américains? La manière directive et exclusive dont les américains ont procédé à l'installation du parc n'a-t-elle pas dissuadé les Samoans d'y participer et de s'y intéresser? Mes observations sur le terrain vont dans ce sens : les populations locales ont, certes, été intégrées au projet dans la forme, mais elles n'ont jamais été réellement consultées, pas plus que leur perception de la nature sollicitée, pour contribuer à l'enracinement du projet dans les objectifs et les préoccupations de la population. Ce constat peut en partie expliquer l'échec du parc: une « étanchéité » de deux points de vue sur la perception de la nature aurait entraîné un échec de toute collaboration entre les deux partenaires. Mais cela n'explique pas tout. L'évolution récente du rapport à la terre chez les Samoans peut aussi éclairer ce problème. Avant de discu-

6. Lors de ma seconde visite aux Samoa américaines, le NPAS avait lancé un projet de lutte contre des espèces invasives « arbres et cochons sauvages » en collaboration avec certains jeunes gens des villages du parc. Selon le responsable de ce programme, la perception du parc et de sa mission changeait progressivement au travers de telles actions et grâce à l'intermédiaire des employés du parc qui diffusaient de l'information à leurs proches dans les villages. Les habitants commençaient donc, selon lui, à prendre conscience du danger de ces espèces pour l'environnement, à comprendre et à respecter le travail des agents du parc, voire même, pour certains, à adhérer davantage au projet de conservation. 
ter ce point, il me faut rappeler la relation que les Samoans entretenaient traditionnellement avec leur environnement naturel et la cosmologie qui l'inspirait.

\section{La relation homme - nature aux Samoa américaines}

À propos des Samoa américaines, le dernier consul anglais pour le Pacifique occidental, William B. Churchward, en poste dans les dernières années de 1880 , écrivait :

«As in Upolu and Savaii, [Tutuila] has a range of mountains running its whole length, with spurs at all angles right down to the seashore, leaving at any spot but little margin for the Samoans to locate their villages. The whole island is densely bushed down to the water's edge, or to the edge of the perpendicular cliffs that every here and there show a considerable drop to the ocean-level. Every little space available, however, is taken up by the native settlements. " (Churchward, $1987: 38)$

Aujourd'hui, la nature et les paysages donnent toujours cette même impression de majesté et de luxuriance. Pourtant, très vite, les bâtiments des deux conserveries et l'énorme station de stockage d'essence nous rappellent que nous ne sommes plus au XIX ${ }^{\mathrm{e}}$ siècle. Les îles ont depuis bien changé et les technologies modernes y sont implantées (toute l'île est reliée au réseau électrique et d'eau potable, l'hôpital est l'un des mieux équipés de la région et les télécommunications y sont des plus modernes). Face à ce contraste entre une nature sauvage d'un côté et une forte industrialisation de l'autre, une question s'impose : comment, aujourd'hui, les Samoans américains perçoivent-ils leur environnement naturel en comparaison de l'attachement personnel et ancestral à la terre dont faisaient preuve leurs ancêtres décrits par les premiers voyageurs et autres anthropologues.

\section{L'attachement des Samoans à leur terre et à leur nature, selon les récits et les textes anciens}

De manière générale, le lien des populations du Pacifique à la terre de leurs îles a été observé et souligné dans toutes les sociétés de la région(Baré, 1992; Bensa, 1992 ; Bonnemaison, 1986 ; Malinowski, 1974 ; Tcherkezoff, 1992). Considérer la terre comme le socle de l'organisation sociale est une «constante culturelle très forte " dans l'ensemble des îles du Pacifique (Bonnemaison 2000 : 521). Jean-François Baré (1992 : 9) parle « d'obsession collective du fait territorial » pour décrire à quel point la question

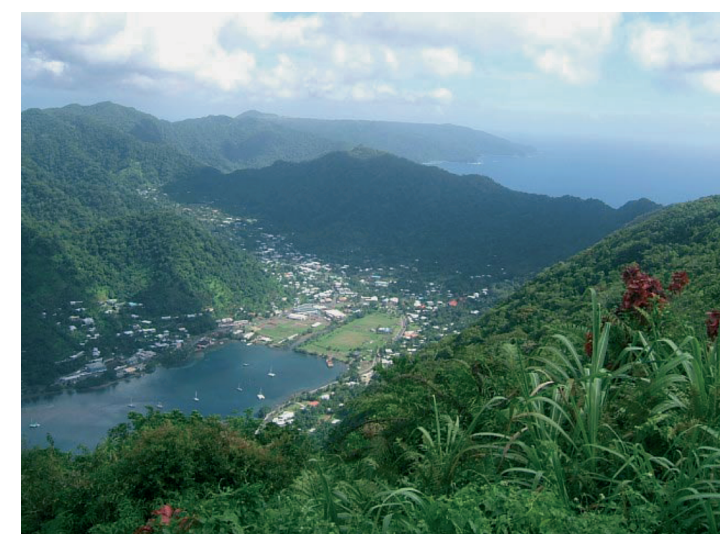

Figure 2. - Baie de Pago Pago vue du haut du Mont Alava (cliché Marieke Blondet, 2006)

de la terre est sensible et centrale dans toute cette région. Cela n'a rien d'océanien. En Israël et en Palestine aussi. L'histoire de la communauté qui a acquis une certaine surface de terre est à l'origine du statut social de cette communauté et de chaque individu en son sein. Groupes et individus n'ont d'identité qu'en relation à la terre. Les Samoans étant à l'origine un peuple venu de la mer, le droit à la terre, à son accès et à sa propriété, a toujours été fondamental pour eux : c'est selon l'ordre d'arrivée dans l'île - puis selon l'issue des guerres internes - que s'est mise en place la hiérarchie des familles et des hommes. D'ailleurs, à Samoa et, par extension aux Samoa américaines, on parle des " enfants de la terre » pour qualifier les habitants des îles (Tcherkezoff, 1992 : 15). La terre y possède une triple dimension : elle est la terre-mère nourricière et fertile qui donne aux hommes leurs moyens de subsistance. Elle est la terre-berceau de l'origine et de l'ancestralité puisque chaque famille étendue (aiga) est liée, à travers son histoire et ses légendes, à un lieu spécifique. La terre est, enfin, la source de l'identité de chaque individu (ibid. : 15). C'est en effet à travers la référence au passé, à l'ancêtre fondateur de la lignée et à la manière dont il a acquis ces terres (en les défrichant, par fait de guerre ou comme récompense de service rendu à un chef de rang supérieur) que tout individu se situe dans l'organisation sociale. Lorsqu'un Samoan se présente, il annonce, avant toute chose, le nom du village dont il est originaire et le nom de sa famille dans ce village. J'ai eu l'occasion d'observer la manière dont des adolescents se présentaient à un exercice scolaire oral. Ils commençaient invariablement leur exposé en disant :

« Je m'appelle $\mathrm{x}$ de la vieille et honorable famille $\mathrm{Y}$ et je vis dans le magnifique village de v. » 
À Samoa comme dans l'ensemble des îles du Pacifique, la place donnée à la terre par les populations et le lien que celles-ci établissent entre cette terre, leurs ancêtres et l'histoire et le nom de leur famille, rendent compte, depuis des temps reculés, de la place de chacun, tant dans l'espace social que dans la possession de la terre. C'est à travers celle-ci qu'un Samoan peut dire et décrire qui il est. C'est du moins ce que l'on peut lire dans la littérature anthropologique sur le sujet. Tel était le constat que pouvait faire un observateur jusqu'au milieu du $\mathrm{XIX}^{\mathrm{e}}$ siècle, environ. Hélas le constat que j'ai pu faire moi-même, entre 2006 et 2007, est tout différent. Voici des éléments permettant d'en juger.

\section{Relations observées entre les Samoans américains et leur environnement}

Lorsque j'ai demandé à des Samoans ce que le terme « nature » signifiait pour eux, ce n'est pas l'image de la forêt luxuriante décrite par Churchward et que je pouvais moi-même observer aujourd'hui qui leur est venue à l'esprit. Leurs yeux se sont tournés vers leurs plantations de bananes et de taros et vers le récif corallien, c'est-à-dire vers les espaces naturels qu'ils utilisent au quotidien.

Fox et Cumberland (1962) avaient déjà pris note de la classification samoane des espaces naturels selon l'utilisation que les hommes en faisaient. Ces auteurs distinguent trois espaces forestiers investis physiquement et symboliquement de manière différente par les Samoans (Fox and Cumberland, 1962:63). Le premier espace est celui de la forêt côtière au sein de laquelle sont implantés les villages et les plantations de cocotiers. Cette forêt a quasiment disparu aujourd'hui. Son emplacement est aussi la partie de l'île la mieux connue des Samoans puisqu'ils y ont implanté leurs villages. La deuxième zone forestière distinguée abrite une forêt de moyenne altitude, qui a été très exploitée pour en extraire $\mathrm{du}$ bois de construction et pour défricher de nouvelles parcelles agricoles. Le troisième espace, enfin, est ce que les auteurs nomment la forêt de montagne et que je qualifierai de forêt d'altitude et de forêt primaire par opposition aux forêts côtières et de moyenne altitude, plus récentes et typiquement secondaires. Cette forêt primaire est la moins exploitée et, partant, la moins connue et donc la plus crainte des Samoans. À propos de cette dernière, Fox et Cumberland remarquent, sans chercher à l'expliquer, que:

«To Samoans this forest area is cold, damp and inhospitable, and is rarely visited except by younger and more venturesome hunters. " (Fox and Cumberland, $1962: 65)$

En effet, à l'exception de quelques chasseurs de cochons sauvages dont la forêt d'altitude est l'habitat habituel, les Samoans ne circulaient pas dans ces zones forestières. À l'inverse, les auteurs précisent que les Samoans étaient particulièrement fiers de la forêt côtière où ils vivaient. Les habitants de chaque village possédant encore une surface de cet espace forestier sur son territoire déclaraient que le lieu possédait les arbres les plus hauts, ceux qui avaient le bois le plus dur ou de la meilleure qualité pour fabriquer des massues de guerre (Fox and Cumberland, 1962 : 73). Cette fierté particulière pour les forêts de basse et moyenne altitude, les plus proches des lieux d'habitation et les plus exploitées, illustre à quel point ces lieux étaient importants pour les Samoans. Ce sont ces lieux qu'ils considéraient comme constituant leur "nature» et non les forêts primaires d'altitude. Une telle distinction de zones spatiales et fonctionnelles est courante dans le Pacifique. Ainsi les communautés kanak de Ponérihouen, par exemple, distinguent les espaces socialisés des hommes, qui comprennent les endroits habités et cultivés ainsi que les zones de pêche, de chasse et de cueillette et un espace surnaturel, dont fait partie la forêt primaire et qui comprend des sites sacrés et des lieux où se pratiquent certains rituels, lieux aussi de résidence des esprits (Leblic, $2005: 100$ ).

Les Samoans américains âgés que j'ai rencontrés m'ont dit avoir encore une relation très étroite avec la nature ou, devrais-je préciser, avec certains espaces naturels particuliers comme nous venons de le voir. Cette relation résultait notamment du fait que la survie de ces hommes et la totalité de leur vie matérielle étaient tributaires des éléments tirés de cet environnement. Fox et Cumberland observent aussi à ce propos:

«From the village land comes, too, the special timber used in building fale [...]. Leaves of the sugar cane, or from the guinea palm, supply thatching materials, pandanus is used for floorard sleeping mates, strips of coconut leaves are woven into blinds and rough mats used in fale or for drying copra and cacao; and the bark of the paper mulberry provides the raw material for siapo [tapa] on which pattern are created with the aid of vegetable dyes. » (Fox and Cumberland, 1962 : 186)

L'ethnobotaniste Arthur Whistler (1996b: 120-131) a, lui, attesté l'utilisation par les Samoans de plantes et de fleurs pour la parure ou la décoration, avant même le contact avec les Occidentaux. Dans une société sensible à la propreté et aux odeurs corporelles, on utilisait les fleurs pour leur parfum et pour parer l'intérieur 
des maisons. À cet effet, des plantes décoratives étaient cultivées autour des habitations et, aujourd'hui, on observe toujours de magnifiques jardins décoratifs autour des maisons. Enfin, au-delà de la simple parure, les médecines traditionnelles sont encore pratiquées et les herbes médicinales utilisées (Whistler, 1996a : 1), même si beaucoup plus rarement que par le passé. Mais c'est, bien entendu, dans l'exploitation agricole des terres que s'établit la relation la plus étroite entre l'homme et la nature et nous allons en voir ci-dessous divers aspects.

\section{Gestion des ressources naturelles et interdits}

Au-delà de ces représentations symboliques de la nature, et pour maîtriser l'imprévisibilité des éléments naturels et s'assurer des ressources constantes, les Samoans ont mis en place, au fil du temps, un système d'exploitation durable de leur environnement. Ils utilisent une méthode de rotation des terres agricoles qui leur permet d'obtenir trois récoltes de taro tous les deux ans sur une même parcelle. Au bout de deux ans, la plantation est abandonnée et une nouvelle surface défrichée. Les Samoans préfèrent ainsi pratiquer des rotations de cultures plutôt que de diversifier leur production agricole. Ce système de rotation des terres était, selon J.W. Coulter (1957), particulièrement adapté à l'environnement naturel des îles car dès qu'une parcelle était abandonnée, elle était immédiatement recouverte de pousses de jeunes arbres. Cette forme d'exploitation était tout à fait adaptée au contexte de faible population du passé. Mais dès le milieu $\mathrm{du} \mathrm{Xx}^{\mathrm{e}}$ siècle, cet abandon à la jachère des parcelles exploitées s'opposait à la poussée démographique qui exerçait une forte pression sur les terres disponibles (Coulter, 1957 : 80-1). La situation est identique aujourd'hui bien que la pression ne se fasse plus sentir sur les terres agricoles, puisque l'activité agricole est largement délaissée par les Samoans américains. La pression s'est transposée sur les terres constructibles.

L'organisation du travail se faisait par les matai qui distribuaient à leurs proches les parcelles de plantation à travailler. Ils répartissaient ensuite le fruit de ce travail entre les membres de leurs familles (Fox and Cumberland, 1962 : 193). Parfois, il arrivait aux matai de promulguer des tabous sur la collecte de telle plante ou sur la pêche de telle espèce marine pour permettre à celles-ci de récupérer d'une exploitation trop intense. Ces tabous existent toujours aujourd'hui et, comme par le passé, sont décrétés par les conseils de villages réunissant l'ensemble des matai de la communauté. J'ai été témoin de certaines de ces décisions. Par exemple, dans le village de Fagasa où, chaque année, au mois d'août, des bancs entiers de maquereaux entrent dans la baie proche du village, moment choisi par la communauté pour organiser une grande pêche collective. L'ensemble des villageois, à pied, encercle les bancs de poissons et les repousse, à l'aide de feuilles de bananier, au plus près du rivage où ils seront attrapés puis partagés entre tous. Cette pêche a toujours existé et donne lieu, comme autrefois, à de grandes réjouissances ; c'est une occasion privilégiée pour resserrer les liens entre les habitants du village. Pour que cette pêche soit la plus fructueuse possible, dès qu'est observée l'arrivée des premiers poissons, les matai émettent une interdiction de pêche valable pour l'ensemble du village, un tabou qui durera jusqu'au jour où le conseil autorisera enfin la pêche collective. D'autres villages pratiquent aussi des interdictions semblables, mais pour d'autres raisons. Encouragé par une initiative gouvernementale du Department of Marine and Wildlife Resources, le conseil du village de Vatia, où j'ai effectué l'essentiel de mon étude, a édicté pendant deux ans une interdiction de pêche de certaines espèces pour en permettre une régénération alors qu'elles avaient jusque-là souffert de surexploitation. Les tabous et restrictions des conseils de villages sont toujours observés aujourd'hui, que ce soit pour respecter une coutume ancienne ou pour protéger des ressources naturelles, encouragés en cela par les mouvements modernes de préservation de l'environnement, attitude pourtant déjà connue des Samoans par le passé.

\section{Les rituels et la relation symbolique à la nature}

Les Samoans avaient donc une gestion précise des ressources naturelles de leurs îles et ils continuent à l'avoir dans certains cas. Cependant, la relation des Samoans américains à la nature allait bien au-delà de la vie matérielle puisque tout l'aspect symbolique et cérémoniel de cette société reposait aussi sur des éléments tenant à la nature. Les nattes de pandanus tressé (fala en samoan), étaient souvent grossières et utilisées dans la vie quotidienne. Mais elles pouvaient aussi être si finement tressées (par les taupou, les filles vierges des plus puissants matai), qu'elles acquéraient une très forte valeur symbolique et étaient rituellement et cérémonieusement échangées en des occasions très particulières. Encore plus symbolique, et directement issue de la nature, la racine de ava (Piper methysticum) était consommée en boisson de manière rituelle dans 
l'ensemble de la région. William Setchell, ethnobotaniste ayant travaillé spécifiquement aux Samoa américaines, explique que les Samoans avaient une idée très précise de la distribution topographique des plantes. Dans leurs mythes, ils montraient une grande connaissance de la succession de celles-ci sur le terrain, ainsi qu'une vision très claire de leur évolution dans le temps (Setchell, 1924 : 218). Ils avaient même développé une nomenclature botanique complexe comme le montre, entre autres, la célèbre chanson de Tutuila racontant la « bataille des arbres et des pierres » et énumérant soixante-dix à quatre-vingts noms d'arbres (Krämer, 1994 : 492). La nature est aussi très présente dans la mythologie où des noms de plantes ont été donnés aux dieux et inversement. De nombreux végétaux ou animaux (oiseaux marins, tortue, requin, anguille) font partie intégrante des mythes de création et sont souvent à l'origine d'améliorations techniques ou de la découverte d'un nouvel aliment. Par exemple, selon le mythe de « Sina et l'anguille », la noix de coco viendrait directement de la tête de l'anguille domestique de la jeune Sina qui aurait enterré, à la mort de l'animal, la tête de celui-ci. De là aurait poussé le premier cocotier. Dans ces mythes, on lit aussi que les hommes sont directement issus des éléments de la nature. Le dieu Tagoloaalagi envoya sur terre un de ses serviteurs, une liane rampante, pour empêcher les arbres de trop grandir et de toucher l'Eden du dieu créateur. Cette liane étant devenue trop luxuriante, le dieu envoya un autre de ses serviteurs pour l'abattre. De cette liane en putréfaction seraient sortis de gros asticots qui, une fois façonnés par un esprit appelé Gaio, donnèrent les hommes (Tala O Le Vavau, 1995 : 99). Enfin, la nature et ses éléments sont aussi très présents dans les proverbes que les Samoans affectionnent tant. Ces dictons utilisent les éléments de la nature comme métaphores pour parler des hommes et de leur relation aux êtres et aux choses qui les entourent. Ainsi un matai de haut rang me cita en exemple le proverbe $O$, o le vao, signifiant « il n'y a rien dans la forêt », et qui sous-entend que si on ne prend pas soin de la forêt, un jour, il n'y aura plus rien dont on puisse dépendre. Cela signifie aussi que si on ne prend pas soin de sa famille, un jour, on risque de se retrouver seul et, comme me l'expliqua ce matai :

"Most samoan proverbs and knowledge are coming from the land and the sea. It tells you all about how important land is. A proverb is to relate what you're doing and what you say to the land and to the sea. Being a chief today, the other chiefs won't look at you if you don't know those things, relating the lands and the sea to what you say. And you have to use them. You know it brings you back to the environment; bring you back to the land itself. » (Extrait d'entretien, 2006)

\section{Croyances anciennes et modernes des Samoans}

La nature, on le voit, était bien plus que la somme d'éléments matériels à exploiter ; elle faisait partie intégrante du symbolisme de la société samoane. De ce fait, dans le passé, les Samoans américains avaient manifestement une forte déférence vis-à-vis de la nature, non seulement pour les raisons que nous venons d'évoquer, mais aussi parce que la partie la plus sauvage et incontrôlée de la forêt primaire était considérée comme le lieu de prédilection des esprits et était crainte, pour cette raison. Cette perception des forêts intérieures comme sites des esprits et du surnaturel se retrouve régulièrement dans certaines îles du Pacifique Sud (Leblic, 2005 : 100101). De nos jours, aux Samoa américaines, si les gens s'excusent presque d'avoir de telles croyances, tant la religion chrétienne a été intégrée, ils échangent pourtant entre eux encore nombre d'histoires et de légendes sur des esprits présents dans cette partie de la forêt. Dans le village où j'ai vécu, les quinquagénaires racontent que, dans leur jeune âge, pour aller à la seule école de l'île située à Pago Pago, ils devaient traverser la forêt d'altitude par un sentier en pente et glissant. Ils disent aussi qu'une petite fille a disparu sur ce chemin et que, depuis, son esprit hanterait une source où les villageois avaient l'habitude de se désaltérer. De nos jours, le peu de personnes qui s'aventurent sur ce chemin et souhaitent boire à cette source, le font très rapidement de peur d'être attirés par l'esprit et de disparaître, comme cela est arrivé à la fillette. J'ai aussi recueilli l'histoire de ces deux femmes archéologues qui, pour gagner du temps sur une fouille, dormirent sur le site en pleine forêt et furent réveillées au milieu de la nuit par des rires tonitruants, sans pouvoir en identifier les auteurs. Les habitants du village à qui appartiennent ces espaces forestiers expliquèrent que le feu qu'elles avaient allumé avait réveillé les esprits des lieux. Cette croyance des Samoans dans les esprits forestiers est ancestrale. William B. Churchward (1987) rapportait en 1880 que, lors d'une marche en forêt, son groupe était arrivé aux ruines d'une guesthouse, maison traditionnelle ouverte dans laquelle les Samoans recevaient leurs visiteurs et dans laquelle les matai du lieu avaient coutume de se rencontrer. Il écrit :

"I heard one very old Samoan say that in times of troubles, or when some important question was to be investigated, the great chiefs would retire there and sit in 
solemn mystical silence, each one with his back against a stone pillar, until one of them should receive inspiration from the spirits supposed to be hovering round the place, and deliver his instructions in oracular form. » (Churchward, $1987: 181$ ).

À part cette note de Churchward, il n'existe, à ma connaissance, aucune autre référence traitant, pour les Samoa américaines, de cette perception surnaturelle de la forêt primaire. Dans un but explicatif et comparatif, je ferai appel aux observations d'Hélène Guiot (2000) à propos de Futuna. En effet, cette île possède de très fortes similarités de culture et de croyances avec les deux Samoa voisines, ce qui permet de faire l'hypothèse que ce qui a été observé à Futuna a aussi pu exister aux Samoa. À Futuna, Hélène Guiot a attesté de l'existence, avant l'arrivée des premiers Occidentaux, de rituels prenant place dans les espaces forestiers les plus denses et ayant pour objectif de s'assurer de la fertilité de la terre. Cette fertilité était, selon les Futuniens, issue du pouvoir de certains dieux vivant au sein de ces espaces forestiers (Guiot, $2000: 9$ ). On dit de la forêt, à Futuna, qu'elle est le ma'uli 'aga o le fenua, le principe vital du pays ${ }^{7}$. Les grands arbres retiennent l'humidité en attirant les nuages puis en conservant l'eau de pluie au contact de leurs racines. Cette atmosphère humide génère de la fertilité pour la terre et procure eau et fraîcheur aux hommes. C'est parce que cette propriété est particulièrement associée au vao matua (les forêts anciennes ou climaciques), qu'autrefois, des rituels de fertilité prenaient place dans cette partie de la forêt. Il faut noter au passage que, soutenant mon hypothèse d'une proximité culturelle forte entre Futuna et les Samoa, le terme de vao matua est également employé aux Samoa pour désigner les forêts les plus anciennes situées au centre de l'île. Toujours selon Hélène Guiot, ces cérémonies reflétaient la volonté des anciens Futuniens de voir prospérer le ulufenua et d'assurer le fonctionnement harmonieux du fenua (Guiot, 2000 : 25). La forêt climacique représentait donc des éléments en équilibre tant symbolique que fonctionnel dans la vie des hommes : ces derniers en tiraient des matières premières, les couples illégitimes et les fuyards s'y réfugiaient et, c'était, surtout, le domaine de prédilection des dieux. Les grands arbres, les vestiges de tombes et les anciens lieux d'habitation constituaient, pour les Futuniens, le siège des temonio, les esprits. Cette croyance est toujours actuelle (ibid.). Associé à cette symbolique de la forêt comme source de toute vie, on trouve le concept de "forêt tabou ", sacrée et donc protégée, concept qui, selon Guiot, est répandu dans toute la Polynésie. De tels rituels de fertilité ont été attestés par des fouilles archéologiques dans d'autres îles de la région (Tikopia, Tonga, Hawaii, Tahiti). Ils l'ont aussi été en Nouvelle-Calédonie (Leblic, 2005 : 105106). Même si rien de tel n'a encore été observé aux Samoa américaines, il est légitime de penser que ces rituels étaient aussi présents ici et que la forêt y avait le même caractère sacré. Les archéologues ont d'ailleurs découvert, au cœur de la forêt primaire, de nombreux star mounds ou tia seu lupe (tumulus en forme d'étoile). Des scientifiques ont proposé que ces structures étaient utilisées pour la chasse aux pigeons mais soupçonnent également, sans avoir encore les éléments pour le confirmer, qu'ils avaient d'autres fonctions rituelles pour la société samoane précontact (Enright, 2005)

L'ensemble de ces hypothèses sur la vie rituelle et symbolique des Samoans américains nous amène à poser la question de savoir si tous ces éléments étaient intégrés à une vision globale du monde, à une cosmologie. Une telle éventualité pourrait alors nous éclairer sur les relations que ces populations entretiennent aujourd'hui avec la nature qui les entoure et, notamment, les attitudes négatives - à nos yeux d'Occidentaux de pollution et de surexploitation qu'ils lui infligent.

\section{Qu'entend-on par cosmologie?}

De nombreuses définitions de ce qu'est une cosmologie ont été données (E. Durkheim, J.G. Frazer, L. Lévy-Bruhl, Cl. Lévi-Strauss parmi d'autres). D'une manière générale, on s'accorde à dire que la cosmologie est la conception que se fait une société de l'univers, c'est-à-dire qu'elle regroupe un ensemble de concepts permettant à cette société d'appréhender le monde autour d'elle (Lévi-Strauss, 1962 : 113). Le discours sur la nature est de l'ordre de la cosmologie puisque la représentation de son environnement naturel par une société associe et fédère l'ensemble des concepts culturels qu'elle a forgés sur le monde. L'importance de cette représentation est cardinale puisque c'est d'elle que procèdent le mode et le droit d'appropriation et d'exploitation des objets naturels (Descola, 1986; Descola and

7. Le terme fenua, que l'on retrouve dans l'ensemble des langues de Polynésie, désigne le pays, l'île dans sa totalité. Le ulufenua est, lui, l'ensemble des entités animées produites par la terre : les végétaux, les animaux, l'eau et l'homme. Enfin le terme ma'uli signifie la fertilité, le principe vital, la vie elle-même source de la croissance et de l'existence de tous les êtres du ulufenua (Guiot, $2000: 9-10)$ 
Palsson, 1996 ; Lévi-Strauss, 1962). La vision du monde, et en particulier de la nature, est par conséquent une construction sociale propre à chaque société (Descola and Parson, 1996 : 15), une conception bornée " par l'horizon d'une certaine vision du monde propre à un certain milieu, à une certaine culture et à une certaine époque » (Berque, 1995 : 36-7). La cosmologie est donc historiquement, localement et socialement circonscrite. Partant de ce constat, Kay Milton pose la question de savoir comment des populations ne parlant pas la même langue et enracinées dans des fonds culturels différents, ayant donc des visions du monde différentes, peuvent arriver à s'entendre et à mettre en place des cadres communs d'actions au profit de l'environnement (Milton, 1993 : 11). L'hypothèse proposée ci-dessus pour rendre compte de l'indifférence des Samoans vis-à-vis des techniques occidentales de remédiation à la surexploitation et à la pollution de leur environnement fournirait une illustration pertinente du point de vue de Kay Milton. Les Samoans ne saisiraient pas les arguments des écologistes occidentaux ou ne percevraient pas les bienfaits des techniques proposées, autrement dit ils seraient imperméables aux recommandations occidentales, parce qu'elles ne trouveraient pas d'écho dans leur propre cosmologie. Une expérience, due à Rachel Turner, soutient cette hypothèse. Cette biologiste marine a travaillé sur la pertinence des programmes d'éducation aux questions d'environnement et de pollution des récifs coralliens aux Samoa américaines. Elle montre à quel point l'information donnée par les écologistes occidentaux ne "passe » pas chez les Samoans et qu'il existe, dans le même temps, de grandes incompréhensions entre eux, pas uniquement du fait de leurs différences de langue d'ailleurs. Selon Turner, les Samoans n'arriveraient pas, à l'évidence, à faire le lien entre leurs pratiques vis-à-vis de l'environnement et les connaissances qui leur sont proposées dans ces programmes éducatifs. En effet les personnes interrogées ne voient pas de relation de cause à effet entre les pratiques comportementales des hommes et l'état de pollution et de grande dégradation des récifs coralliens (Turner, 2005). Pour trouver les raisons de cette dissociation peut-être faut-il aller chercher plus loin, dans l'étude de la cosmologie samoane, la manière dont cette société pense la nature et la relation qu'elle entretient avec elle. Ceci pourrait expliquer la source de l'incompréhension entre Samoans et écologistes américains et fonder l'espoir que l'intégration de ces connaissances pourrait, à terme, déboucher sur l'adoption de pratiques appropriées à la pro- tection de l'environnement aux Samoa américaines. Quelle est donc la vision du monde et de la nature des Samoans américains?

\section{Fa'asamoa : une cosmologie samoane}

Aux Samoa, pour qualifier leur manière de voir le monde, les gens utilisent le mot de fa'asamoa. Ce terme désigne tout à fois l'ensemble de ce qui a trait à la culture, la façon de faire samoane et la représentation du monde samoane. Nous avons déjà noté l'attachement à la terre des Samoans : terre nourricière, féconde et donc sacrée. Une représentation idéelle qui, au-delà, a donné l'aspect matériel de la société samoane, ses modes d'exploitation de l'environnement et son organisation sociale fondée sur la possession, ou son absence, de la terre. Les rituels tels ceux de fertilité observés à Futuna et très probables à Samoa, évoqués ci-dessus, en sont également un témoignage. Cependant la cosmologie samoane va bien au-delà de ce seul paramètre qu'est le rapport à la terre. On trouve aussi, au centre du faa'samoa, le groupe familial, l'aiga, avec à sa tête un senior matai, choisi par consensus des membres de la famille pour la représenter publiquement et agir pour son bien. L'appartenance à une famille offre un accès privilégié à un certain nombre de terres qui lui sont réservées. Les membres de l'aiga ont, en contrepartie, des devoirs et des obligations vis-à-vis de leurs proches et de leur matai, comme ceux de participer à la vie du groupe et de la partager. Ces devoirs et ces obligations sont, de fait, des fondements de l'appartenance familiale et permettent à chacun de se situer dans l'espace social. Mais cette description est réductrice et n'éclaire pas la part de la relation des Samoans à leur cosmogonie dans leurs réactions et dans leurs attitudes vitales et sociales profondes. Serge Tcherkézoff (2003) a tenté d'identifier la vision du monde des Samoans et, compte tenu du passé historique et culturel commun aux deux Samoa, il est justifié de penser que ce qu'il a décrit s'applique de manière quasiment identique aux Samoa américaines. De nombreux chercheurs considèrent les Polynésiens comme des navigateurs dont la vision du monde est tournée vers l'océan. Cependant, pour Serge Tcherkézoff, les Polynésiens, notamment les Samoans ont, pour appréhender le monde, une "philosophie de la lumière ». L'auteur fonde son argument sur les récits des premiers Occidentaux dans les îles qui ont rapporté que le soleil était le signe de la vie chez les Polynésiens préchrétiens et qu'un véritable culte lui était porté. Tcherkézoff explique que toute la vision de l'espace des Samoans est orga- 
nisée autour de ce culte solaire : l'est, le lieu du lever de l'astre solaire, est le lieu de l'espoir, du futur, de la vie, alors que l'ouest est le lieu des « origines historiques », de la mort, des ancêtres et des esprits malins (2003: 36-37). D'ailleurs nombre de mythes situent le royaume des morts et/ou l'île d'origine mythique à l'ouest. Cette organisation mentale de l'espace expliquerait que la conquête des îles et de l'océan Pacifique ait eu lieu d'ouest en est, en direction du soleil. Un mythe des îles Cook expliquant le peuplement de l'île d'Aitutaki illustre cette analyse :

«Deux frères habitaient le "pays de l'obscurité" [L'ouest, là où le soleil se couche et disparaît]. Ils s'élancèrent sur la mer, persuadés qu'en allant suffisamment loin ils trouveraient "la terre de la lumière" [Vers l'est]. » (ibid. : 39)

Serge Tcherkézoff va plus loin et reconnaît ce dualisme est-ouest dans les oppositions entre jour et nuit, lumière et obscurité et même jusque dans la géographie humaine et l'installation des villages. Ces derniers constitueraient des cercles sacrés avec, en leur centre, un espace vide, le malae, que le soleil peut inonder. Dès qu'on s'éloigne de ce centre, du côté des terres, on avance vers des espaces plus obscurs, plus incontrôlables, plus sauvages :

« Vers l'intérieur des terres, ce sont les abris pour la cuisine, puis les jardins, plus loin les plantations au centre de l'atoll ou sur les pentes des montagnes des hautes îles, puis, dans ce dernier cas, la forêt. Vers la mer, c'est le lagon, ensuite le récif et enfin la haute mer. » (Tcherkézoff, $2003: 45)^{8}$

Dans ce modèle, on le voit, la forêt primaire de l'intérieur des îles est du côté de l'obscur : ces lieux sont naturellement sombres puisque les grands arbres empêchent le passage de la lumière. Cet espace est aussi vécu comme incontrôlable car c'est l'endroit où les esprits vivent et circulent. La forêt primaire fait donc partie, dans l'esprit des Samoans, du pôle obscur, surnaturel, et donc dangereux, en opposition au pôle lumineux et rassurant du village et de la communauté. Ce point de vue est repris par Alfred Gell qui explique que la cosmologie polynésienne repose sur la séparation originelle, par le dieu fondateur, du ciel et de la terre, du jour et de la nuit. Cette séparation aurait alors constitué le modèle d'un monde bipolaire divisé entre, d'un côté, le po, «l'autre monde», celui de la nuit (po), des dieux originels et de la mort, et de l'autre, le monde ao de la lumière, du jour ( $a o)$, de la vie, de l'activité humaine (Gell, 1995:
21-23). La différenciation originelle entre ces deux pôles appartenant à un même monde serait l'archétype de la vision polynésienne du monde : d'un côté le pôle lumineux, organisé, où s'élaborent les relations entre les hommes et l'attribution des positions statutaires - c'est là que se constitue une société socialisée, de l'autre, le pôle obscur, éloigné des activités sociales. L'homme y est livré à lui-même et aux éléments surnaturels, c'est l'espace du chaos.

Aujourd'hui, presque plus personne aux Samoa américaines ne parle en ces termes symboliques, d'une part parce que la religion chrétienne y est très influente, d'autre part parce que les Samoans se considèrent trop "modernes" pour croire au surnaturel. Pourtant, les théories de Tcherkézoff et de Gell sont intéressantes et renforcent les hypothèses que $\mathrm{j}$ 'ai formulées à partir de mes propres observations. On peut en effet penser qu'il reste dans la mémoire collective des Samoans des éléments de ces croyances anciennes qui interviendraient encore dans leur appréhension des forêts primaires qui les entourent. Cet espace fait partie, dans la cosmologie samoane, du pôle surnaturel po, inquiétant et non porteur d'espoir. Cette observation expliquerait donc la distance que les Samoans américains conservent par rapport à la forêt intérieure. Ils se tiennent à l'abri de toute ingérence et interférence avec cet espace aux mains de forces surnaturelles incontrôlables et, par conséquent, comprennent mal que les Américains du parc national puissent vouloir protéger ces espaces. La réaction des Samoans conduit à une protection naturelle relative du parc, puisque par désintérêt et crainte, la population n'investit pas, de quelque façon que ce soit, ces espaces forestiers. Cet état d'esprit contraste totalement avec la conduite observée vis-à-vis du $a o$, l'espace de lumière et de vie : celui que l'on plante, dans lequel on circule, celui où naissent les liens sociaux. Les tentatives des écologistes américains pour convaincre la population locale de la nécessité de protéger la nature mériteraient d'emprunter beaucoup plus à la cosmologie samoane. Mais il est vrai que le succès de ces tentatives repose sur celui, rédhibitoire, de la pénétration des forêts du parc par les insulaires.

En regard de ce qui se passe dans les forêts primaires, les Samoans américains fréquentent quotidiennement leur $a o$, espace de vie et de lumière. Qu'y observe-t-on? Une surexploitation de leurs ressources et une pollution systématique de leur environnement. Le recours à la perception du monde des Samoans américains

8. Les espaces marins, s'ils ne sont pas à proprement parler sombres, n'en sont pas moins obscurs car ils sont tout aussi inconnus et incontrôlables que les forêts primaires. Des puissances surnaturelles y circulent aussi. 
ne donne pas d'explication convaincante au caractère destructeur de leurs comportements vis-à-vis de leur espace $a o$. Partant de ce constat, une seconde hypothèse pour expliquer l'attitude négative de la population de ces îles vis-à-vis de l'écosystème de l'espace $a o$, serait que leur vision ancestrale du monde ne trouverait plus la même résonance chez eux. Comment alors expliquer la perte d'efficacité manifeste de la cosmologie samoane? L'évolution récente du rapport des Samoans américains à leurs terres de famille peut, en partie sans doute, aider à l'expliquer.

\section{La relation samoane aux terres familiales}

Mes observations, en 2006 et en 2007, m'ont montré qu'il est extrêmement difficile de faire parler un Samoan de sa relation à la terre ; tout au plus est-il capable de discuter des terres de sa famille, de les situer autour du village, voire de les montrer physiquement. Je n'ai rencontré personne parlant de la terre dans des termes symboliques en relation à son identité et à la place qui est la sienne dans la société, à l'image de ce qui a été décrit par d'autres dans le Pacifique. Au contraire les personnes que j'ai interrogées m'ont parlé de leurs terres comme d'une « propriété de famille », voire d'un «capital familial ». Il se pourrait alors que la perception de la terre ait glissé d'une symbolique à l'autre. Si la relation terre / identité individuelle existe toujours, puisque c'est l'appartenance à une aiga, associée à un ancêtre fondateur qui lui a donné son nom et son titre de matai qui fixe l'identité de chacun, le fait de parler des terres familiales en termes de "propriété ", même s'il s'agit d'une propriété communautaire, semble indiquer une rupture du lien qui unissait les hommes à la terre et, par extension, à la nature. Les matai - et même les «métayers »-doivent avoir encore un intérêt à ce que leurs terres soient entretenues, fertilisées... Tout au moins, un chaînon important manque dans l'appréhension des terres que cultivent les Samoans américains d'aujourd'hui, celui du symbolisme attaché aux terres familiales.

Aux Samoa américaines, la terre est un bien communautaire qui appartient à l'ensemble du groupe familial sans qu'aucun membre ait plus de droits que d'autres sur ce bien. Les véritables possesseurs de ces terres sont les ancêtres de l'aiga. Les membres vivants de la famille, eux, exploitent ces terres familiales et en sont les garants pour les générations futures. Les pouvoirs coloniaux ont conservé ce système d'organisation ancestral et la législation des Samoa américaines relative à la terre et à la propriété foncière est un mélange de droit américain et de coutume samoane. Il en résulte qu'aujourd'hui les terres sont toujours majoritairement sous le régime communautaire bien que la notion de propriété privée soit connue et de plus en plus acceptée. Le senior matai de chaque aiga est responsable de ces terres de famille. Il est responsable de leur maintien dans les meilleures conditions possibles et ses proches se reposent entièrement sur lui pour tout ce qui concerne la gestion de ces biens. Personne n'est donc totalement propriétaire, ni totalement responsable, des terres aiga, à part le matai qui, selon la coutume, est d'avantage le gérant que le propriétaire de ces espaces. Cette situation peut dès lors expliquer pourquoi, aux Samoa américaines, et malgré les programmes éducatifs, les habitants continuent à avoir des comportements irresponsables et polluants vis-à-vis de la nature. L'hypothèse que je propose est qu'ils continuent de le faire justement parce que les terres sur lesquelles ils vivent ne leur appartiennent pas en propre mais qu'elles sont celles des ancêtres fondateurs. Ces derniers, autrefois très respectés, ont perdu de leur primauté dans la vie sociale des Samoans depuis l'adoption du christianisme. Parler de la terre de ses ancêtres n'a plus le même sens que jadis ; aujourd'hui, lorsqu'un Samoan invoque ses ancêtres, il remonte rarement au-delà de ses arrières, arrières grands-parents et il aura davantage tendance à parler de la terre de sa famille étendue dans le présent, que de la terre de ses ancêtres. En outre, les Samoans savent très bien ce qu'est la propriété privée et font tout à fait la différence entre celle-ci et la « propriété aiga » communautaire. Dans une société où les personnes s'individualisent toujours plus et tendent chaque jour un peu plus vers un style de vie "à l'américaine ", libérés de trop fortes obligations familiales, cette propriété individuelle est une tentation forte. Parallèlement, les Samoans ayant conscience que leurs terres de famille ne sont pas vraiment leurs, se déresponsabiliseraient vis-àvis de ces espaces et, au final, n'auraient pas de scrupules à jeter leurs déchets dans la nature, même si la terre concernée appartient à leur famille, et avec moins de scrupules encore si cette terre est celle du voisin et/ou celle du village. Ainsi assiste-t-on à des scènes comiques entre des femmes qui, tous les matins, dès les premières lueurs de l'aube, balayent consciencieusement l'espace devant leur maison (celui-ci se doit, traditionnellement, d'être toujours impeccable de propreté) alors que la veille, elles avaient jeté mégots de cigarettes et papiers de bonbons chez leurs voisines, qui leur avaient rendu la pareille. 
Au-delà de ces attitudes, l'attachement coutumier à la terre s'est lui aussi progressivement délité, à l'image de la désaffection pour les activités agricoles et la pêche. Cette perte de sens des terres aiga, et de leur appartenance au groupe familial et à ses ancêtres, est particulièrement forte dans la plaine de Tafuna où les occupants louent, majoritairement, les terres sur lesquelles ils habitent et que, de fait, quasiment plus aucune référence aux relations entre la généalogie d'une famille et une terre ne transparaît. Cet endroit est celui de l'île qui se développe le plus vite, où se concentre la plus large population et où l'on défriche le plus pour construire. C'est aussi l'endroit de Tutuila où l'on pollue le plus. Au final, la question qui se pose est celle du sens de la responsabilité des personnes: on prend instinctivement la responsabilité d'une terre possédée individuellement, mais pas d'une terre aiga ne vous appartenant pas en propre. On ne pollue pas un bien privé, mais celui des membres de sa famille étendue sur lequel on a des droits d'usage, mais pour lequel on ne peut décider comme on le ferait pour un bien privé. Aux Samoa américaines, on fait donc face à une double irresponsabilité : d'une part, parce que la terre n'est le bien propre d'aucun des membres de l'aiga. D'autre part, parce que c'est le matai qui en a la charge. Cette irresponsabilité est évidemment encore plus forte lorsqu'il s'agit de terres appartenant à l'état, à une autre aiga ou à un propriétaire privé auquel on n'est pas apparenté. La notion de responsabilité vis-à-vis d'un bien propre ne semble pas être familière aux Samoans américains. Et si la propriété privée se développe de plus en plus aux Samoa américaines, la responsabilité associée à la propriété individuelle, notamment d'objets naturels, n'a pas encore été intégrée.

Ces idées ont déjà été discutées par de nombreux chercheurs, notamment Garret Harding (1968) qui a développé la célèbre théorie de la «tragédie des communs». L'argument de cet auteur est que les ressources détenues de manière collective seront à long terme irrémédiablement détruites car aucun individu isolé n'a de motivation suffisante pour prendre la responsabilité de ces ressources. Cependant, cette théorie ne rend pas entièrement compte de la complexité de la situation que j'ai observée aux Samoa américaines. Harding part en effet du principe que des ressources détenues collectivement sont forcément des ressources n'appartenant à personne. Elles sont donc en « accès libre » et à la disposition de tous et se trouvent vite surexploitées dès lors que chaque utilisateur place son intérêt personnel immédiat avant celui de la communauté toute entière. Jamais Harding ne considère la possibilité d'une gestion communautaire de ces ressources (Berkes and Farvar, 1989: 7) et sa théorie ne prend pas en considération, à l'évidence, les biens détenus par un groupe comme l'étaient et le sont, pro parte, les terres aiga aux Samoa américaines. Je préfère, pour cette raison, adopter le point de vue de Berkes et Farvar pour qui la propriété d'un bien communautaire est gérée de manière communautaire (Berkes and Farvar 1989 : 8). L'exemple samoan, où la répartition du travail puis des produits de celui-ci entre les membres de l'aiga est organisé sous l'autorité du matai, correspond à cette situation. Cet exemple parmi d'autres prouve que les utilisateurs de ces ressources communes peuvent coopérer et, de fait, le font. Ces ressources détenues de manière communautaire ne sont évidemment pas accessibles aux personnes n'appartenant pas au groupe. L'accès aux terres est régulé à l'intérieur et à l'extérieur. Pour revenir à cet état de déresponsabilisation de la population samoane vis-à-vis de sa terre, je serais tentée de conclure que cette situation est le résultat de facteurs historiques qui ont affecté l'attachement et le respect des Samoans pour leur terre et, au-delà, pour la nature : d'une part, l'adoption d'un style de vie à l'américaine avec une consommation de plus en plus importante de biens manufacturés dont les déchets, chimiquement résistants à la décomposition polluent l'environnement de façon durable et devant lesquels les Samoans ne peuvent avoir qu'une attitude passive avec l'acceptation progressive de l'idée de propriété privée et la disparition progressive du magistère des ancêtres et du respect qui leur était dû. Ces paramètres, en lien avec les développements récents de la théorie des communs, peuvent, selon moi, apporter une explication valable à la dégradation de l'environnement par la population. La surexploitation, c'est encore autre chose.

\section{BIBLIOGRAPHIE}

American Samoa's Comprehensive Economic Development Strategy, 2005. Pago Pago (American Samoa), Department of Commerce.

BARÉ Jean-François, 1992. La Terre, le Pacifique. Introduction, Études rurales 127-128, pp. 9-14.

Bensa Alban, 1992. Terre Kanak: Enjeu politique d'hier et d'aujourd'hui, Études rurales 127-128, pp. 107-131.

BERKES Fikret and M. Taghi FARVAR, 1989. Introduction and Overview, in Fikret Berkes (éd.), Common Property Resources. Ecology and Community-based Sustainable Development, London, Belhaven Press. 
Berque Augustin, 1995. Les raisons du Paysage. De la Chine antique aux environnements de synthèse, Paris, Éditions Hazan.

Bonnemaison Joël, 1986. La Dernière île, Paris, oRsTOM.

—, 2000. Océanie, in Pierre Bonte et Michel Izard, Dictionnaire d'Ethnologie et Anthropologie, Paris, PUF, collection Quadrige, pp. 521-522.

Bryant-Tokalau Jenny, 2004. Governance of Poverty and Environment in the Pacific: Where are the experts?, Sites 1, 2, Asaanz ed., pp. 25-56.

Butcher Russel, 1999. Guide to National Parks, Pacific Region, Guilford (Connecticut), National Parks and Conservation Association, The Globe Pequot Press.

Churchward William B., 1987 (first published 1887). My Consulate in Samoa, Southern Reprints, London, Richard Bertley and Son.

COLCHESTER Marcus, 1994. Salvaging Nature: Indigenous Peoples, Protected Areas and Biodiversity Conservation, Genève, UNRISD.

Coulter John Welsey, 1957. The Pacific Dependencies of the United States, New York, The Mac Millan Company.

Craig Peter, 2005 ( $2^{\text {nd }}$ édition). Natural History Guide to American Samoa, Ed. National Park of American Samoa, Dept. Marine and Wildlife Resources of American Samoa and American Samoa Community College.

DesCola Philippe, 1986, La nature domestique. Symbolisme et praxis dans l'écologie des Achuar, Paris, Éditions de la maison des sciences de l'homme.

Descola Philippe and Gisli Palsson, 1996. Nature and Society: Anthropological Perspectives, London, Routledge.

ENRIGHT John, 2005. The Past Surrounds Us, Part 7: Star Mounds, site Internet de l'American Samoa Historic Preservation Office.

Fox James W. and Keneth B. Cumberland, 1962. Western Samoa. Land, Life and Agriculture in Tropical Polynesia, Christchurch (New Zealand), Whitcombe \& Tombs Ltd.

Gell Alfred, 1995. Closure and Multiplication: An Essay on Polynesian Cosmology and Ritual, in Daniel de Coppet and André Iteanu (eds), Cosmos and Society in Oceania, Berg Publisher Limited.

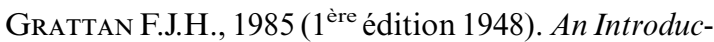
tion to Samoan Custom, New Zealand, McMillan Publisher.

Gray J.A.C., 1980. Amerika Samoa, New York, Arno Press.

Guiot Hélène, 2000. Gestion traditionnelle des espaces forestiers à Futuna : contenu idéel et pratiques associées, Journal de la Société des Océanistes 110, pp. 19-33.

Harding Garret, 1968. The Tragedy of the Commons, Science 162, American Association for the Advancement of Science Publisher, pp. 1243-48.
HefFer Jean, 1995. Les États-Unis et le Pacifique, Histoire d'une frontière, Paris, Albin Michel.

Holmes Lowell D. and Ellen RhoAds Holmes, 1992 ( $1^{\mathrm{e}}$ éd. 1974). Samoan Village. Then and Now, Fort Worth (USA), Harcourt Brace Jovanovich College Publishers.

HuFfer Elise and Ropate QALO, 2004. Have we been thinking upside-down? The Contemporary Emergence of Pacific Theoretical Thought, The Contemporary Pacific 16, 1, University of Hawaii Press, pp. 87-108.

KRÄMER Augustin, 1994, The Samoan Islands, translated by T. Verhaaren, Vol.1, Pasifika Press, Auckland, New Zealand. (First publication 1902, Die Samoa-Inseln. Einwurf einer Monographie mit besonderer Berücksichtigung Deutsch-Samoas (2 vol., 1902-1903), E. Schweizerbartsche Verlagsbuchhandlung (E. Nägele), Stuttgart.

LEBLIC Isabelle, 2005. Pays, "surnature» et sites " sacrés " paicî à Ponérihouen (Nouvelle- Calédonie), Journal de la société des Océanistes 120-121, pp. 95-111.

LÉvi-Strauss Claude, 1962. La Pensée Sauvage, Paris, Plon, Collection Agora Pocket.

Malinowski Bronislaw, 1974. Les jardins de corail, Paris, Maspero.

Milton Kay, 1993. Introduction, in Milton Kay (ed.), Environmentalism. The view for anthropology, London, Routledge.

-, 1996. Environmentalism and Cultural Theory. Exploring the role of Anthropology in environmental discourse, London, Routledge.

National Park of American Samoa. General Management Plan: Environmental Impact Statement, 1997 (oct.). United States Department of the Interior, National Park Service publication (personal communication).

O’Meara Tim, 1990. Samoan Planters. Tradition and Economic Development in Polynesia, Fort Worth (USA), Holt, Rinehart and Winston Inc.

OverTon John, 1999. Sustainable Development and the Pacific Islands, in OverTon John and Regina Scheyvens (eds.), Strategies for Sustainable Development. Experience from the Pacific, Australia, University of New South Wales Press, pp. 1-15.

Public Law 100-571, 1988 (October 31).

Scheyvens Regina and Nick Purdie, 1999. Ecotourism, in Overton John and Scheyvens Regina (eds), Strategies for Sustainable Development. Experience from the Pacific, Australia, University of New South Wales Press, pp. 212-226.

Setchell William A., 1924. American Samoa: Part II - Ethnobotany of the Samoans, Dpt. of Marine Biology of the Carnegie Institution of Washington, vol.xx.

SHAFFER Robert J., 2000. American Samoa. 100 Years Under the United States Flag, Hawaii, Island Heritage Publishing. 
SogGE David, 2004 (septembre). Une nécessaire réforme de l'aide internationale, Le Monde diplomatique, p. 10.

TALA O LE VAVAU. Myths, Legends and Customs of old Samoa, 1995 ( $1^{\text {st }}$ published 1976). Auckland, Pacifika Press.

TCHERKÉzOFF Serge, 1992. Les enfants de la Terre aux îles Samoa : tradition locale et « développement » importé, Études rurales $127-128$, pp. 15-40

_, 2003. Faa-Samoa, une identité polynésienne, Paris, L'Harmattan.

Turner Rachel, 2005. An Assessment of Public Perception for the Planning of Environmental Education, communication personnelle.
Wells Mickael and Katrina Brandon, 1992. People and Parks, Linking Protected Area Management with Local Communities, Washington DC, The World Bank.

Whistler Arthur, 1996a. Samoan Herbal Medecin, Honululu, Isle Botanica Editor.

-, 1996b. Plants in Samoan Culture, the Ethnobotany of Samoa, Honululu, Isle Botanica Editor.

WiLson Alexander, 1992. The Culture of Nature. North American Landscape from Disney to Exxon Valdez, Cambridge (USA), Blackwell. 
